\title{
Collaborative Drug Therapy Agreement Topics in the State of Washington: From Dispensing Pills to Managing Ills
}

\author{
Nancy M Hecox* \\ Clinical Supervisor, Tieton Village Drug, Yakima WA, USA
}

Received: June 22, 2014, Accepted: July 08, 2014, Published: July 22, 2014

*Corresponding author: Nancy M Hecox, Clinical Supervisor, Tieton Village Drug, Yakima WA, USA, E-mail: nhecox@gmail.com

\section{Introduction}

In 1979, Washington State pharmacists were granted dependent prescriptive authority via collaborative drug therapy agreements with any practitioner licensed in the state of Washington authorized to prescribe legend drugs.

Collaborative drug therapy agreements (CDTAs) in accordance with the laws (RCW 18.64.011) [1] and regulations (WAC 246-863-100) [2] allow pharmacists prescriptive authority in various settings and for various purposes. There is no statutory authority to limit the pharmacists' prescriptive authority to any particular class of drugs or location of practice. The intent of the CDTA regulation is to increase the capacity and access to pharmaceutical care for the citizens of WA State so that health outcomes are improved.

A CDTA is a prepared, written guideline or protocol algorithm authorizing the pharmacist to include specific prescribing functions into their practice. The CDTA document may include prescribing for the management of disease states, medication refills, preventative health care, medication reviews and emergency situations.

\section{Purpose}

This paper provides a description of the current CDTA topics on file with the Washington State Department of Health credentialing department.

Having reviewed hundreds of CDTAs, it is possible to categorize the list into practice settings, noting that the CDTA itself could stand in any desired setting. This paper includes a sample of CDTAs, as not all of the protocols had expired and were in need of renewal.

\section{Retail and Independent Pharmacy CDTAs}

Immunization protocols include VFC (Vaccine for Children) [3], adolescent and adult routine preventative care, travel shots and free vaccine for adults (VFA). VFA is an additional collaborative effort with Merck $^{\circledR}$ Vaccine and Sanofi ${ }^{\circledR}$ vaccine. The Merck Patient Assistance Program (MV-PAP) melds a pharmacy, a local health department and Merck $^{\circledR}$ who provides free vaccine for eligible adults that are uninsured or under insured [4].
Sanofi ${ }^{\circledR}$ provides free vaccine and supplies via the three-way collaboration between the pharmacy, local health department and Sanofi ${ }^{\circledR}$ through a program called Americares ${ }^{\circledR}[5]$.

Additional protocols that fit with a travel clinic model include Malaria prophylaxis, Traveler's diarrhea treatment, and Motion sickness prevention and treatment.

Prenatal vitamin prescriptions, ECP, Opiate overdose (naloxone) nasal spray, Tuberculosis testing, Pink Eye testing and treatment, H. Pylori testing and treatment, Rapid Strep testing and treatment are additional protocols in effect at retail stores. It is possible for a pharmacist to provide simple emergency medical services as a revenue stream at the pharmacy level especially in rural areas with limited medical access in addition to the distribution of medication, MTM services and preventative health screenings.

\section{Hospital System Pharmacists and Closed Clinic System CDTAs}

System pharmacists are likely to be exclusively engaged in CDTA prescriptive authority with no distribution activities. The protocols that follow include initiation of dosing, adjusting dosing, reviewing and reconciling medications, discontinuing medications, providing antidotes and emergency medication responses where needed, usually per accepted AHRQ medical guidelines or algorithms. Pharmacists order lab tests and adjust doses per physical examination as well, setting appointments with patients independent of a physician visit.

Additional protocols include Anticoagulation clinics; Aminoglycoside dosing; Total Parenteral Nutrition (TPN) management and compounding; Hospice and Palliative Care; Diabetes, Hypertension and Lipid management; Vancomycin dosing; Renal dosing; HIV therapy management; Anemia management; Transplant medication management; Mental Health management (depression, schizophrenia, bipolar, mania, addiction) and Seizure management including seizure kits.

Hospice and Palliative Care can be further broken down into several sub-protocols that include Alteration in skin integrity, Anxiety, Bowel care (constipation and diarrhea), 
Cough, Depression due to end of life issues, Dyspnea, Elevated temperature (fever), Eye care, Heartburn/reflux/belching, hiccups, Itching (pruritus), IV line care, Narcotic induced sedation, Nasal congestion, Nausea and Vomiting (opioid/narcotic related nausea), Nutrition, Oral care (candida, mucositis, herpes/cold sores, cancer sores/stomatitis, dry mouth), Pain, Pediatric pathways, Perianal pain (candidiasis, hemorrhoidal, perineal discomfort), Sedation for comfort, Sleep pattern disturbance, Terminal care, Thrombocytopenia, and Urinary symptoms (bladder spasms/infection, urinary incontinence).

Many hospital and clinic systems rely on a team of pharmacists to refill medications for system patients, as this popular protocol exists for most systems in Washington State.

As of July 1, 2014, the state of Kansas joined the other 48 states and Washington, D.C in a nationwide trend towards advancing the practice of pharmacists who wish to utilize their doctor of pharmacy (Pharm D) degrees to improve drug therapy and healthy outcomes for their patients [6].

In contrast pharmacists in the UK under the National Health System have two avenues for prescriptive authority as supplementary prescribing (SP) introduced in 2003 or independent prescribing (IP) introduced in 2006 [7]. Supplemental prescribing and collaborative drug therapy agreements are generally equivalent practices with American pharmacists moving to advance practice degrees and specialties as the health care system environment changes. The UK offers an advanced practice degree deemed the Masters Advanced Pharmacy Practice degree (pgdip/msc) that makes a pharmacist qualified to be a Pharmacist Independent Prescriber [8].

\section{Conclusion}

The current practice of clinical pharmacists in the State of Washington reflects a professional practice at the level of dependent prescriptive authority. Pharmacists are valued members of a team of providers taking the role of medication specialist providers. Insurance companies recognize this value as prescriptions written by pharmacists are covered entities. Furthering pharmacist's credibility into prescribing can be supported by a comparable advanced master's degree offered to our UK counterparts.

For twenty three years, Washington State has recognized the value of pharmacist's interventions via the CDTA regulations.
There are approximately 6300 registered in- state pharmacists who also provide advanced patient-centered services such as coordination of medications during care transitions, medication management, comprehensive medication reviews with ongoing medication monitoring, chronic disease management, disease education, prevention and wellness services, and patient education [9]. For patients to achieve the full benefit of their medications, pharmacists must be part of the health care team as pharmacy practice advances from distributing pills to managing ills.

Further outcome research concerning the impact in patient care would be the next interesting step as pharmacists take responsibility for medication selection, monitoring and management.

\section{References}

1. Washington State Legislature. RCW 18.64.011 Definitions. [Cited 2014 May 05]; Available from: (http://apps.leg.wa.gov/rcw/default. aspx?cite $=18.64 .011$ )

2. Washing State Legislature. WAC 246-863-110 Monitoring of drug therapy by pharmacists. [Cited 2014 May 05]; Available from: http:// apps.leg.wa.gov/wac/default.aspx?cite=246-863-110

3. CDC. Vaccine for Children Program. [Cited 2014 May 05]; Available from: http://www.cdc.gov/vaccines/programs/vfc/index.html

4. Merck Patient Assistance Program. [Cited 2014 May 05]; Available from: http://www.merckhelps.com/MPAP/

5. Americares.org. Medical Outreach. [Cited 2014 May 05]; Available from: http://www.americares.org/global-health/medical-outreach/

6. American Pharmacists Association. Kansas collaborative practice becomes law July 1. [Cited 2014 May 07]; Available from: http:// www.pharmacist.com/kansas-collaborative-practice-law-becomeseffective-july-1-1

7. Tonna AP, Stewart D, West B, McCaig D. Pharmacist prescribing in the UK - a literature review of current practice and research. J Clin Pharm Ther. 2007; 32(6):545-56.

8. Robert Gordon University Aberdeen. Masters Advanced pharmacy Practice Degree. [Cited 2014 May 07]; Available from: http://www. rgu.ac.uk/health-professions/study-options/part-time-learning/ advanced-pharmacy-practice

9. American Pharmacists Association. Provider status: What pharmacists need to know now. [Cited 2014 May 05]; Available from: http://www. pharmacist.com/provider-status-what-pharmacists-need-know-now 